\title{
REFERENCES
}

HOCKING, The Language Laboratory in the USA (in Jalling (ed.) Modern Language Teaching, London 1968) p. 51

ORNSTEIN and LADO, Research on Foreign Language Teaching Methodologj', IRAL V/1

VALDMAN, The Implementation and Evaluation of a Multiple-Credit Self-Instructional Elementary French Course, Bloomington, 1965

ADAMS, A Proposed Computer-Controlled Language Laboratory' (in Jalling (ed.) Modern Language Teaching, London, 1968) p. 68

ABOUT THE AUTHOR: Dr. Jalling is Director of English Studies and Director of the Language Lab Research Project, University of Stockholm, SWEDEN

\section{LANGUAGE RECORD DISTRIBUTORS}

The following list of record distributors is in no way a complete listing; but is intended only to provide a service to interested NALLD members.

In order to compile a more complete listing we ask members who know of additional names and addresses to send them to the NALI.D Publications Center.

Ariola-Eurodisc-German; opera, operatic recitals, lieder, operettas, Baroque and chamber music, choral and sacred works, drama, poetry, documentaries, dance and cocktail music, vocal hits, evergreens, marches and folk music. West Germany.

Bremen House, Inc.-German; popular, fairy tales and lullabies, alpine music. 218 East 86th St., New York, N. Y. 10028.

Caedmon Records, Inr.-English, Elvish, French, German, Greek, Japanese, Russian, Spanish, Yiddish; American and English literature, drama, folksongs, children's records, social studies, Biblical and religious, art and architecture. 5058 th Ave., New York, N. Y. 10018 . 
Capitol Records Distributing Corp.-Imports from Algeria, Arabia, Argentina, Armenia, Austria, China, Denmark, England, Egypt, France, Germany, Holland, India, Ireland, Italy, Japan, Mexico, Philippines, Scotland, Spain, Turkey, Yugoslavia; classical and popular mainly, but has all other subjects and types of records. 1750 North Vine St., Hollywood, Calif. 90028 or: 1290 Ave. of Americas, New York, N. Y. 10019.

Children's Music Center, Inc.-African, Alaskan, Canadian, French, German, Indian (Asia), Indian (America), Japanese, Latin American, Mexican, Spanish; rhythms, rhythm instruments and bells, music listening and appreciation, stories and language arts, social studies, sing along, skills for exceptional children, physical education, science and nature, folksongs, square dance, singing and party games. 5373 West Pico Boulevard, Los Angeles, Calif. 90019.

Curriculum Materials Center-Folk, French, German, Latin American, Spanish; language teaching through songs. 5128 Venice Boulevard, Los Angeles, Calif. 90010.

Doter Publications, Inc.-French, German, Greek, Hebrew, Italian, Japanese, Portuguese (European), Russian, Spanish (American); listen and learn records. 180 Varick St., New York, N. Y. 10014.

EMC Corporation-French, German, Hebrew, Spanish; popular, dialogues, comprehension tests, life of the people (all records are teaching records). 180 East Gth St., St. Paul, Minn. 55101.

Irolku'aj's/Scbolastic Records-Africa, Aran Hebrides, Asia and Middle East, Australia, Brittany, Canada, Caribbean, Central America and Mexico, Eskimos, France, Greece, Hungary, Indian (American), Italy, Lithuanian, Malta, Negro (American), Netherlands, New Zealand, Norway, Pacific Islands, Portuguese, Rumania, Russia, South America, Spain, Turkey, Ukraine, Yugoslavia, U. S. A.; folksongs mainly, poetry, literature. 906 Sylvan Ave., Englewood Cliffs, N. J. 07632.

Forzano and Fleri, Inc.-Italian; San Remo Festivals (popular), instrumental, opera, folklore, poetry, orchestra. 128 Mulberry St., New York, N. Y. 10013. 
Moniter (Music of the World)-Africa, Armenia, Australia, Austria, Baltics, Bulgaria, Chile, China, Czeck, England, Estonia, France, Georgia, Germany, Greece, Gypsy, Holland, Hungary, Iceland, Ireland, Israel, Italy, Japan, Korea, Latin America, Latvia, Lebanon, Lithuania, Mexico, Middle East, Philippines, Poland, Portugal, Rumania, Russia, Scandanavia, Scotland, South America, Spain, Sweden, Russia, Ukraine, U. S. A., Yugoslavia, Etc.; folk and popular recordings. 156 Fifth Ave., New York, N. Y. 10010.

Nieil A. Kjos Music Company-French, German, Spanish: song books and sing along recordings for modern language study. 525 Busse, Park Ridge, Ill. 60068.

Orfeo Importing Company-English, French, German, Italian, Spanish; literature, listen and learn language records, native spoken records, classical, junior science, poetry, drama. $350 \mathrm{~S}$. Goodman St., Rochester, N. Y. 14607.

Spoken Arts, Inc.-American, Chinese, English, French, German, Hebrew, Italian, Irish, Russian, Spanish; poetry, drama, Shakespeare, children's stories and poems, tales and humor, Belles Lettres (essays and speeches), science, folk. 59 Locust Ave., New Rochelle, N. Y. 10801.

The Spoken IW ord, Inc.--English, American; Shakespeare mainly, literature, drama, poetry, essays, history, letters, features, speeches, debates, hearings, readings, discussions, fables. 180 Varick St., New York, N. Y. 10014.

Publisbers Central Bureau (Turn About Vox)-English, European; Classical Records. 33.20 Hunters Point Avenue; Long Island City, N. Y. 11101.

Thomas J. Valentino, Inc. (Major Records)-African, English, European, Latin American, Oriental, Western and Indian (American); mood music mainly, action, religious, dance, comedy, cartoon, industrial, sounds, jazz, children's, romantic, etc. 150 West 46 th St., New York 36, N. Y.

$V$ anguard Recording Society, Inc.-American, English, European; classical, Bach Guild Recordings, folk blues, popular, entertainments, experimental. 71 West 23rd St., New York, N. Y. 10010.

Wilmac Recorders-French, German, Italian, Russian, Spanish; Language Learning Records: "Circling the Globe with Speech," dialogues, pronunciation drills, literature. 921 East Green Street, Pasadena, Calif. 
Goldsmith's Music Shop, Inc.-French and Spanish only; Teaching songs: sing along, Christmas songs; poetry mainly, drama, popular, extracts from books, classic theatre, children's records, folk, novels, excerpts from de Gaulle's speeches, classical readings, theatre of the Middle Ages, critical essays, instrumental, scientificeconomical records, comedy, journalism, painting and art, history, philosophy and religion, tales and stories, language instruction. Language Dept., 401 West 42nd St., New York, N. Y. 10013.

Houghton Mifflin Company-English, French, German, Spanish; records as teaching aids: poetry, literature, plays, patterns of conversation. 110 Tremont St., Boston, Mass. 02107.

Kapp Records, Inc.-American Country and Western, English, French, German, Greek, Hawaiian, Italian, Jamaican, Russian, South American; instrumental, folksongs from all countries, popular, film tracks, children's records, dance music, contemporary music mainly. 136 East 57th St., New York, N. Y. 10022.

Librairie De France, lnc.-French; popular, classical, educational, military music, poetry, plays, children's music, Christmas songs, advanced studies in literature and diction. French Building, Rockefeller Center, 610 Fifth Ave., New York, N. Y. 10020.

London Records, Int--American, English, French, German, Italian, Russian, Spanish, etc. (International Recordings); popular, opera, classics, spoken word, folk, instrumental. No Address.

Lorraine Music Co.-French, German, Greek, Latin, Russian, Spanish; children's records, folksongs, documentary, popular, operas, operettas, theatre, poetry, prose, language instruction. P. O. Box 4131, Long Island City, N. Y. 11104.

Lyric Sales, Inc.-French, German, Greek, Hebrew, Italian, Japanese, Latin, Russian, Spanish; teaching records, plays, prose, poetry (all spoken-very little music). No Address.

Lyrichord Discs, Inc.--German, Spanish; Music of the 16th and 17th Centuries, Music of the Middle Ages. 141 Perry St., New York, 14, N. Y.

Mielke-German; popular, folk, dance, instrumental, children's records, classicil. 242 East 86th St., New York, N. Y. 10028. 